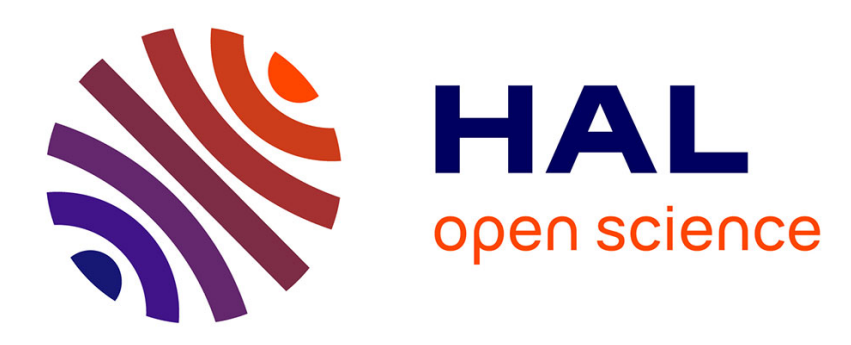

\title{
Étude par photocapacité des centres profonds dans Gap
}

\author{
G. Vincent, D. Bois, J.J. Marchand, P. Pinard
}

\section{To cite this version:}

G. Vincent, D. Bois, J.J. Marchand, P. Pinard. Étude par photocapacité des centres profonds dans Gap. Revue de Physique Appliquée, 1976, 11 (3), pp.393-396. 10.1051/rphysap:01976001103039300 . jpa-00244073

\section{HAL Id: jpa-00244073 https://hal.science/jpa-00244073}

Submitted on 1 Jan 1976

HAL is a multi-disciplinary open access archive for the deposit and dissemination of scientific research documents, whether they are published or not. The documents may come from teaching and research institutions in France or abroad, or from public or private research centers.
L'archive ouverte pluridisciplinaire HAL, est destinée au dépôt et à la diffusion de documents scientifiques de niveau recherche, publiés ou non, émanant des établissements d'enseignement et de recherche français ou étrangers, des laboratoires publics ou privés. 


\title{
ÉtUde PAR PHOTOCAPACITÉ DES CENTRES PROFONDS DANS GaP
}

\author{
G. VINCENT, D. BOIS, J. J. MARCHAND et P. PINARD \\ Laboratoire de Physique de la Matière (*), Institut National des Sciences Appliquées de Lyon, \\ 69621 Villeurbanne, France
}

(Reçu le 3 septembre 1975, révisé le 28 novembre 1975, accepté le 15 décembre 1975)

\begin{abstract}
Résumé. - Nous rapportons les résultats de mesures photocapacitives effectuées sur des échantillons de GaP obtenus par épitaxie vapeur ou liquide et par L. E. C. En plus de l'oxygène qui apparaît dans $\mathrm{GaP}$ vapeur, deux défauts ont été détectés. Ils introduisent des niveaux situés à $1,5 \mathrm{et} 1 \mathrm{eV}$ respectivement au-dessous de la bande de conduction et ont été observés dans des matériaux préparés en phase liquide.
\end{abstract}

\begin{abstract}
We report photocapacitance measurements made on GaP samples obtained by vapour or liquid epitaxy or by L. E. C. In addition to oxygen, which appears in GaP vapour, two other defects were detected. They introduce energy levels at 1 and $1.5 \mathrm{eV}$ below the conduction band and were also observed in materials prepared from the liquid phase.
\end{abstract}

1. Introduction. - Le rendement quantique des diodes électroluminescentes au phosphure de gallium est, en général, limité par des recombinaisons non radiatives sur des défauts. Des études de T. S. C. (courant stimulé thermiquement) ont récemment montré qu'une corrélation existait bien entre la présence de certains pièges et le rendement des dispositifs [1]. En outre, les défauts affectent non seulement le rendement, mais aussi la dégradation des diodes $[1,2,3]$. Il est donc utile de recueillir le maximum d'informations expérimentales sur ces défauts.

Dans GaP, de nombreuses études ont été réalisées en T. S. C. [1] [4-6] ; elles ont permis de mettre en évidence plusieurs défauts, mais la T. S. C. est limitée à l'observation de pièges dont l'énergie d'ionisation est inférieure à $1 \mathrm{eV}$ environ. De plus, cette méthode, lorsqu'elle est utilisée sur des barrières de Schottky, ne permet de mettre en évidence que les pièges à porteurs majoritaires. D'autres techniques expérimentales ont été utilisées, mais principalement pour l'analyse de l'oxygène dans GaP, il s'agit de la photoluminescence [7-10], de la photocapacité $[11,12]$ et de l'absorption optique $[13,14]$ et plus récemment de mesures capacitives [15].

Nous rapportons, ici, les résultats de mesures photocapacitives effectuées sur du GaP de provenances très diverses (épitaxies liquide et vapeur, massif). On sait que cette méthode permet l'observation de pièges sans limitation d'énergie et qu'elle est sensible à tous les types de pièges, même avec des barrières de Schottky.

La technique expérimentale utilisée a été décrite

Ce travail a été soutenu par la D. R. M. E.

(*) Equipe de recherche associée au C. N. R.S. précédemment [16] : elle consiste à étudier les variations de la capacité d'une jonction polarisée en inverse lorsque celle-ci est soumise à un flux lumineux monochromatique. Pour une barrière de Schottky, sur un semi-conducteur de type $N$, la capacité est donnée par :

$$
C=A\left(\frac{\varepsilon q}{2 V} N^{+}\right)^{1 / 2}
$$

$N^{+}$: concentration des charges fixes dans la zone désertée, $A$ : surface de la diode, $V$ : somme du potentiel interne et du potentiel appliqué.

L'émission d'électrons vers la bande de conduction par absorption de photons se traduira donc par une augmentation de $C$ et l'émission de trous vers la bande de valence par une diminution de $C$. La sensibilité des mesures capacitives permet de détecter des variations de $N^{+}$de l'ordre de $10^{12} \mathrm{~cm}^{-3}$. Ceci reste évidemment valable pour une jonction p-n. En outre, les variations de capacité doivent être inversées dans le cas des matériaux de type $\mathrm{p}$.

2. Résultats expérimentaux. Discussion. - Nous avons étudié une série d'échantillons monocristallins obtenus par différentes méthodes : épitaxie en phase vapeur, en phase liquide, double épitaxie et L. E. C. (Liquid Encapsulated Czochralski). Les barrières de Schottky étaient réalisées par évaporation d'or sur la surface décapée du semi-conducteur. Pour les échantillons provenant d'une double épitaxie (une couche $p$ déposée sur une couche $n$ ), nous avons directement utilisé l'interface n-p comme jonction d'étude. Les spectres de photocapacité obtenus entre 0,6 et $2,5 \mathrm{eV}$ peuvent être classés en trois catégories. 
2.1 EPITAXIE eN PHASE VAPeUR. - La figure 1 montre un spectre typique obtenu à $77 \mathrm{~K}$ dans des conditions de quasi-équilibre. L'état initial en début de spectre est défini par l'illumination de la diode en

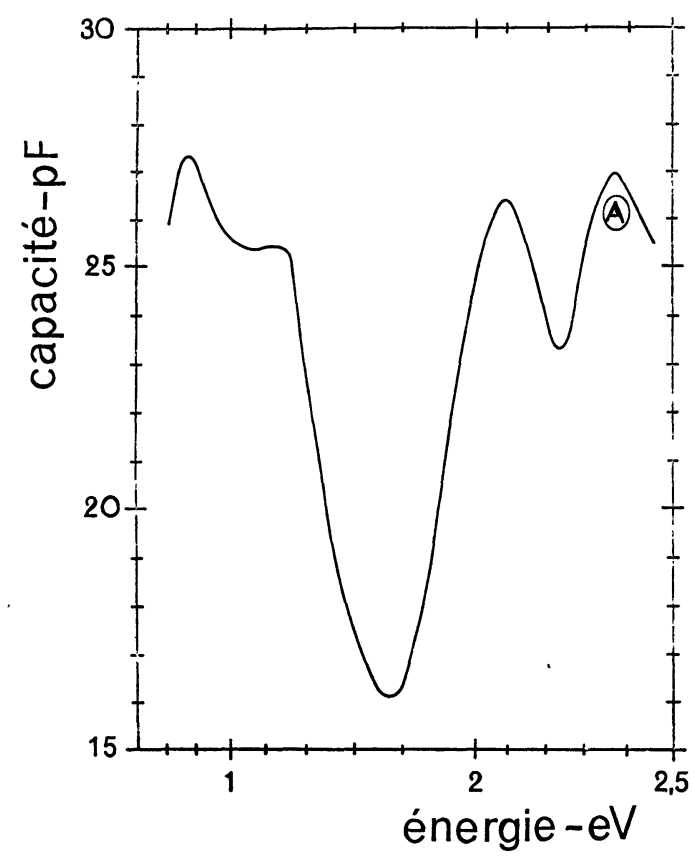

Fig. 1. - Spectre de photocapacité $\mathrm{C}(\mathrm{h} v)$ à $77 \mathrm{~K}$ d'une barrière de Schottky réalisée sur un échantillon de GaP préparé par épitaxie vapeur. Polarisation de la diode $V=-2 \mathrm{~V}$.

lumière blanche. On note trois bandes principales (autour de 0,$8 ; 2,2$ et $2,4 \mathrm{eV}$ ) correspondant à l'émission d'électrons (augmentation de la capacité) et un minimum centré sur $1,8 \mathrm{eV}$ qui traduit l'émission de trous.

Nous avons observé, en outre, qu'une polarisation de la barrière dans le sens direct conduit à un remplissage des niveaux. En effet, si après une telle polarisation maintenue pendant quelques secondes, on illumine la diode à $\mathrm{h} v=1,8 \mathrm{eV}$, on n'observe sensiblement aucune variation de la capacité. Par contre, le vidage induit à $\mathrm{h} v=0,8$ ou $2,1 \mathrm{eV}$ peut être totalement restauré par une illumination à $1,8 \mathrm{eV}$. Ceci démontre que ces bandes sont complémentaires, c'est-à-dire qu'elles correspondent à des transitions sur les mêmes niveaux énergétiques : transitions vers la bande de conduction pour les vidages et depuis la bande de valence pour le remplissage.

Toutes ces observations peuvent s'interpréter en considérant que ces échantillons contiennent de l'oxygène. En effet, Kukimoto et Henry [11, 12] par l'étude de $\mathrm{GaP}$ dopé à l'oxygène, ont observé que cette impureté introduit deux niveaux énergétiques correspondant à deux états d'ionisation possible de l'oxygène substitutionnel. Ces auteurs trouvent les maximums de sections efficaces optiques à environ $1,1 \mathrm{eV}$ et $2,1 \mathrm{eV}$ pour l'émission d'électrons, et à $1,8 \mathrm{eV}$ pour celle des trous. On constate un bon accord pour les deux bandes de plus haute énergie. Par contre, il existe un décalage inexpliqué pour le vidage du premier état $(0,8 \mathrm{eV}$ dans nos mesures au lieu de $1,1 \mathrm{eV}$ ).

Sur la figure 1, on observe aussi la préférence d'un maximum secondaire à $1,3 \mathrm{eV}$. Un tel maximum secondaire, également visible dans les résultats de Kukimoto [11], pourrait correspondre aux transitions de l'oxygène vers le minimum $\Gamma$ de la bande de conduction. Le premier, à plus basse énergie, doit traduire les transitions vers le minimum $X$ qui sont plus probables par suite de la masse effective plus forte des électrons en $\mathrm{X}$ [17]. L'écart de $0,5 \mathrm{eV}$, observé entre les deux maximums, est en bon accord avec la séparation $\Gamma-\mathrm{X}: 0,54 \mathrm{eV}[17,18]$.

De l'amplitude de la variation de capacité mesurée à $\mathrm{h} v=2,1 \mathrm{eV}$, on peut déduire une estimation de la concentration en oxygène dans ces matériaux. Par exemple, l'échantillon présenté figure 1 contient environ $5 \times 10^{15}$ at $0 / \mathrm{cm}^{3}$, ce qui constitue une compensation importante puisque $\left(N_{\mathrm{D}}-N_{\mathrm{A}}\right)$ vaut ici environ $10^{16} \mathrm{~cm}^{-3}$ (valeur calculée à partir de la capacité dans l'obscurité à $300 \mathrm{~K}$ ).

Le dernier pic (A) visible sur la figure 1 correspond très vraisemblablement au vidage des accepteurs hydrogénoïdes. Il existe, plus ou moins bien résolu, sur tous les échantillons. Le seuil de la transition se situe vers $2,25 \mathrm{eV}$, ce qui correspond assez bien à $\left(E_{\mathrm{g}}-E_{\mathrm{A}}\right)$ puisque $E_{\mathrm{g}}=2,32 \mathrm{eV}$ et $E_{\mathrm{A}}$ (énergie de liaison des accepteurs) est compris dans $\mathrm{GaP}$ entre 50 et $200 \mathrm{meV}$ suivant la nature de l'accepteur $[18,19]$.

2.2 Simple ÉPITAXIE LiQUide et LEC. - La figure 2 représente un second type de spectre, obtenu sur des échantillons préparés par épitaxie liquide ou LEC. On note successivement un remplissage (C), puis un

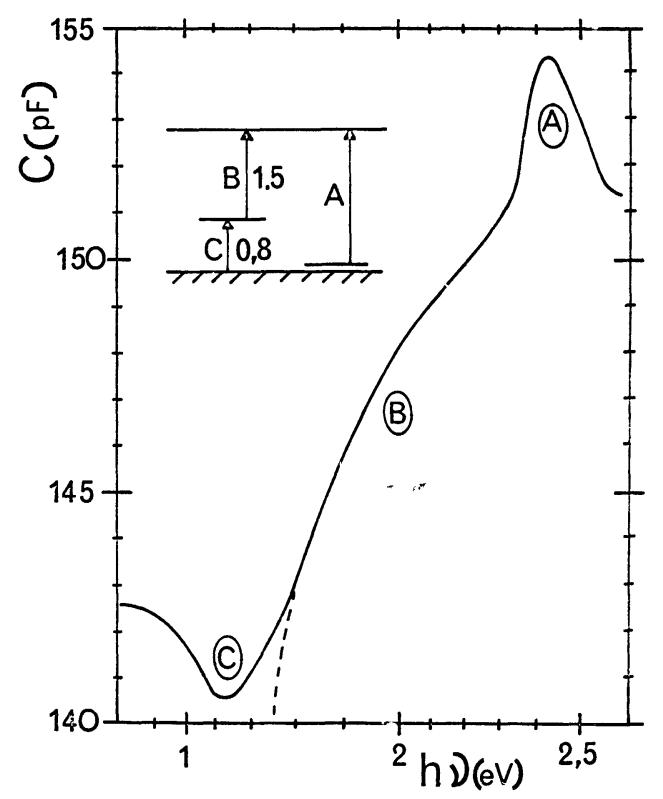

Fig. 2. - Spectre de photocapacité à $77 \mathrm{~K}$ d'une barrière de Schottky sur du GaP obtenu par épitaxie liquide. $V=-0,4 \mathrm{eV}$. Les énergies en $\mathrm{eV}$ indiquées dans l'encadré pour $\mathrm{B}$ et $\mathrm{C}$ sont celles des seuils de transition obtenus par extrapolation. 
vidage (B) de centres. La figure 3 montre que ces deux effets sont complémentaires. Dans ces dernières expériences, la diode était d'abord soumise à un éclaire-

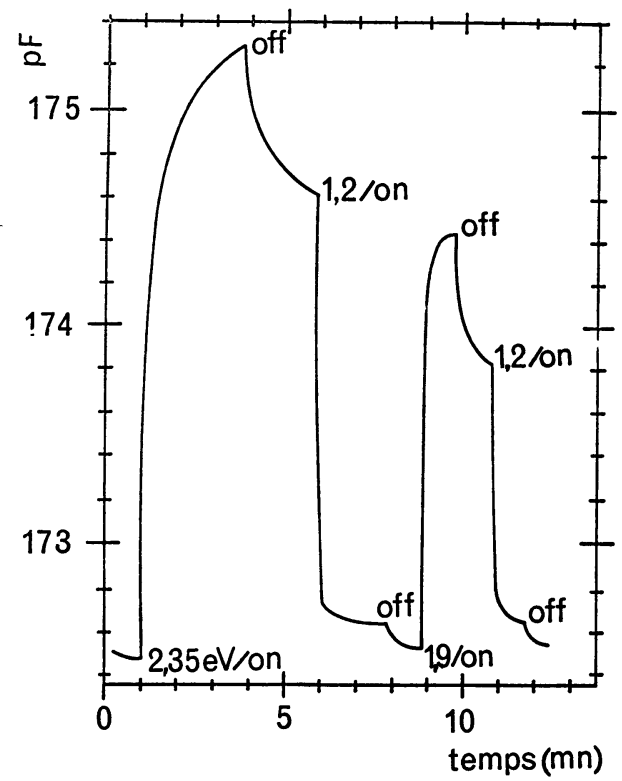

Fig. 3. - Variation de la capacité en fonction du temps au cours de cycles d'éclairement à différents $\mathrm{h} v . T=77 \mathrm{~K}, V=-1 \mathrm{~V}$. Même échantillon que figure 2.

ment correspondant à un vidage $\mathrm{h} v=2,35 \mathrm{eV}$ puis, après un temps de ralentissement de la dérive sous obscurité, soumise à un éclairement à $\mathrm{h} v=1,2 \mathrm{eV}$, valeur correspondant au remplissage (C) visible sur la figure 2. On constate que l'éclairement à $1,2 \mathrm{eV}$ restaure rapidement la capacité. Les effets plus lents observés sous obscurité traduisent un remplissage thermique des centres [19].

Finalement, la courbe de la figure 2 peut s'interpréter en considérant la présence, dans ces matériaux, d'un seul défaut profond. Comme il a été discuté précédemment, dans le cas de GaAs [16] [20,21], il est difficile de définir la position exacte d'un tel niveau par rapport aux bandes d'énergie à cause des phénomènes de couplage avec le réseau qui élargissent les bandes d'absorption, et donc de photocapacité. Cependant, si on élimine par extrapolation les queues des transitions, on peut déterminer des valeurs approximatives des seuils d'ionisation optique du niveau considéré. Ici, on trouve (cf. Fig. 2) respectivement $0,8 \mathrm{eV}$ pour les transitions $\mathrm{BV} \rightarrow$ niveau et $1,5 \mathrm{eV}$ pour niveau $\rightarrow \mathrm{BC}$.

Nous avons recherché, dans la littérature, si un tel niveau avait déjà été observé. Olsson [13] montre que le cuivre introduit des niveaux situés entre 0,64 et $0,8 \mathrm{eV}$ de l'une des bandes, sans pouvoir dire s'il s'agit de bande de conduction ou de bande de valence. Caruso [7] observe une émission en photoluminescence autour de $1,2 \mathrm{eV}$ dont il ne précise pas l'origine. Dishman [22] et Hamilton [23] enfin rapportent l'existence de deux centres profonds situés à 0,4 et $0,7 \mathrm{eV}$ respectivement de la bande de valence. Dishman discute l'origine de ces niveaux et émet l'hypothèse qu'il s'agit de complexes du type $\mathrm{V}_{\mathrm{Ga}}: 3 \mathrm{D}_{\mathrm{P}}$ (centre à $0,4 \mathrm{eV}$ de la bande de valence) et $\mathrm{V}_{\mathrm{Ga}}: 2 \mathrm{D}_{\mathrm{p}}$ (centre à $0,7 \mathrm{eV}$ de la bande de valence). $D_{P}$ symbolise, ici, un donneur en position phosphore dans le réseau. Cette dernière étude a été faite avec des échantillons de GaP dopés au tellure ou au soufre provenant de croissance en solution ou de L. E. C. c'est-à-dire identiques à ceux envisagés dans ce travail. On peut donc penser que le centre que nous avons observé provient de l'un des complexes cités plus haut. Mais il n'est pas possible d'éliminer complètement la responsabilité éventuelle du cuivre. Notons que récemment Hamilton [24] a également observé, dans GaP obtenu par épitaxie liquide, un centre à $0,75 \mathrm{eV} \pm 0,05$ au-dessus de la bande de valence. Il ne discute pas son origine, mais montre que ce centre a une influence déterminante sur la durée de vie des porteurs minoritaires et sur le rendement radiatif dans GaP.

Comme pour l'oxygène, on peut estimer la concentration de ce défaut : $10^{15} \mathrm{~cm}^{-3}$ pour l'échantillon présenté sur la figure 2 .

2.3 Double éPITAXIE LIQUIDE. - La figure 4 montre une troisième allure de spectres de photocapacité, rencontrée dans les échantillons préparés par double

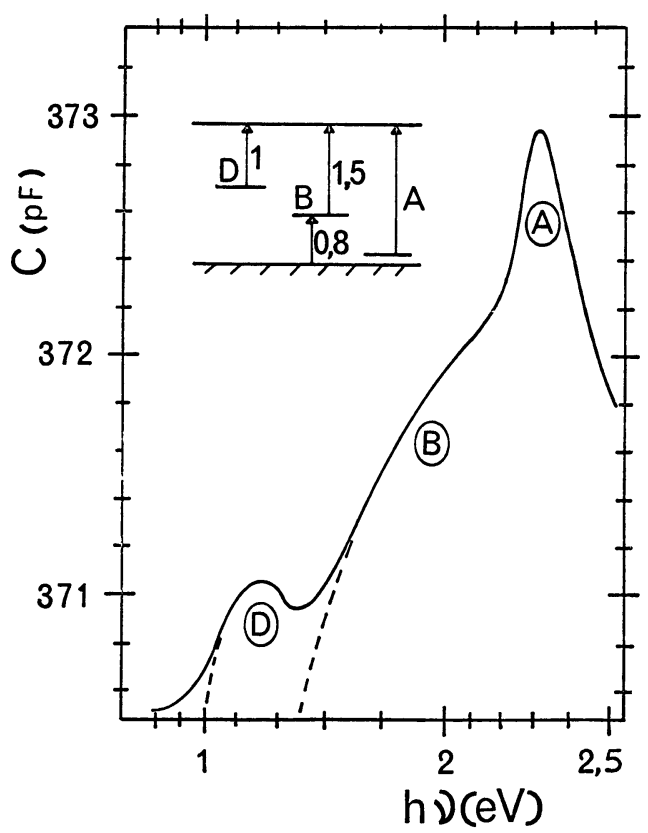

Fig. 4. - Spectre de photocapacité à $77 \mathrm{~K}$ d'une diode GaP obtenue par double épitaxie liquide. $V=-1 \mathrm{~V}$. Les énergies indiquées en $\mathrm{eV}$ pour $\mathrm{B}$ et $\mathrm{D}$ sont celles des seuils de transition.

épitaxie liquide. La jonction étant abrupte côté $\mathrm{p}$, nous supposerons que les phénomènes observés se déroulent dans la zone $\mathrm{n}$ comme précédemment pour les Schottky.

Il apparait, en plus des accepteurs hydrogénoïdes (A), une large bande autour de $2 \mathrm{eV}$ (B), puis une autre aux environs de $1,3 \mathrm{eV}$ (D). Toutes les deux correspondent à l'émission d'électrons. La première peut, du moins en 
première analyse, être assimilée à la bande $\mathrm{B}$ observée dans les échantillons de simple épitaxie (cf. Fig. 2). Le minimum que l'on devrait alors trouver à $1,2 \mathrm{eV}$ puisque $B$ et $C$ sont complémentaires, est ici masqué par un autre niveau (D) dont le seuil se situe à environ $1 \mathrm{eV}$. En effet, dans ce type d'échantillon, il est normal que l'on retrouve les phénomènes visibles dans les matériaux obtenus par simple épitaxie puisque la couche $n$ est identique dans les deux cas. Le niveau supplémentaire (D) semble, par contre, introduit au cours de la croissance de la couche $p$. On peut penser qu'il s'agit d'un défaut se trouvant à l'interface n-p, c'est-àdire dans la zone active de la jonction. Grimmeiss [25], par des mesures de photocourant dans des jonctions, a trouvé un niveau à $\left(E_{\mathrm{c}}-0,98 \mathrm{eV}\right)$ qui pourrait donc être le même que celui que nous observons, mais cet auteur ne discute pas l'origine de ce défaut.

3. Conclusion. - Cette étude expérimentale met en évidence la présence dans le phosphure de gallium de deux niveaux profonds introduits par des défauts dont les concentrations atteignent quelques $10^{15} \mathrm{~cm}^{-3}$. Le premier (noté $B$ dans le texte) a été détecté dans des échantillons (barrière de Schottky) obtenus par simple épitaxie liquide ou par L. E. C. Son seuil d'ionisation optique avec émission d'électrons se situe à $1,5 \mathrm{eV}$, et celui correspondant à l'émission de trous à $0,8 \mathrm{eV}$ environ. Pour le second niveau (noté D), rencontré dans des diodes réalisées par double épitaxie liquide, le seuil d'émission d'électrons se trouve à environ $1 \mathrm{eV}$. Enfin, il a été montré que de l'oxygène peut être introduit accidentellement avec des concentrations de quelques $10^{15} \mathrm{~cm}^{-3}$ au cours de la croissance du GaP en phase vapeur.

Ces résultats complètent les analyses de T. S. C. ou de photoluminescence largement appliquées à la caractérisation de GaP. Il reste à entreprendre une étude physique détaillée de chacun des centres dont nous avons montré l'existence. Pour cela, il faudra déterminer les énergies d'activation thermique ainsi que l'allure des variations spectrales des sections efficaces d'absorption optique, afin de préciser quel modèle de transitions doit être appliqué. En outre, la mesure des sections efficaces de capture des deux types de porteurs devrait permettre de préciser le rôle que peut jouer chacun de ces centres sur les propriétés électriques et les rendements de luminescence de GaP.

Remerciements. - Les auteurs remercient vivement Mme A. Gallais (L. E. P.), MM. J. P. André (L. E. P.) et D. Diguet (R. T. C., Caen) qui ont bien voulu fournir les échantillons nécessaires à cette étude, et Mme Y. Baudet pour son assistance technique dans la préparation des diodes.

\section{Bibliographie}

[1] Fabre, E., Bhargava, R. N., Appl. Phys. Lett. 24 (1974) 322.

[2] IKoma, T., OKumara, T., ESSDERC Conf. 1974.

[3] Bergh, A. A., I. E. E. E. Trans. Electron. Devices, ED 18 (1971) 166.

[4] Fabre, E., Bhargava, R. N., Zwicker, W. K., J. Electron. Mater. 3 (1974) 409.

[5] Smith, B. L., Inst. of Phys. Conf. Ser no 22 (1974) 210.

[6] Smith, B. L., Hayes, T. J., Peaker, A. R., Wight, D. R., Appl. Phys. Lett. 26 (1975) 122.

[7] Caruso, R., Didomenico, M., Verleur, H. W., von Weida, A. D., J. Phys. Chem. Solids 33 (1972) 689.

[8] Dishman, J. M., Phys. Rev. B 3 (1971) 2588.

[9] Dean, P. J., Henry, C. H., Phys. Rev. 176 (1968) 928.

[10] Bhargava, R. N., Review article in Philips Tech. Rev. 32 (1971) 261.

[11] Kukimoto, H., Henry, C. H., Merritt, F. R., Phys. Rev. B 7 (1973) 2486.

[12] Henry, C. H., Kukimoto, H., Miller, G. L., Merritt, F. R., Phys. Rev. B 7 (1973) 2499.
[13] Olsson, R., Phys. Stat. Sol. 46 (1971) 299.

[14] Olsson, R., J. Phys. Chem. Solids 33 (1972) 549.

[15] Lang, D. V., J. Appl. Phys. 45 (1974) 3014.

[16] Bors, D., Boulou. M., Phys. Stat. Sol. (a) 22 (1974) 671.

[17] Dean, P. J., Prog. Solid State Chem. (Pergamon-Press) 1973, vol. 8.

[18] VINK, A. T., Thesis. Eindhoven (1974).

[19] Dean, P. J., Frosch, C. J., Henry, C. H., J. Appl. Phys. 39 (1968) 5631.

[20] Bors, D., J. Phys. (F) C 3 (1974) 241.

[21] Bois, D., Pinard, P., Phys. Rev. B 9 (1974) 4171.

[22] Dishman, J. M., Daly, D. F., Knox, W. P., J. Appl. Phys. 43 (1972) 4693.

[23] Hamilton, B., Inst. of Phys. Conf. Ser. no 22 (1974) 218.

[24] Hamilton, B., Peaker, A. R., Bramwell, S., Harding, W., Wight, D. R., Appl. Phys. Lett. 26 (1975) 702.

[25] Grimmeiss, H. G., Inst. of Phys. Conf. Ser., no 22 (1974) 187. 